\title{
ANIMISMO, ARTIFICIALISMO E FINALISMO NEL PENSIERO PREOPERATORIO
}

\author{
Neala Ambrosi-Randić \\ Università Juraj Dobrila di Pola \\ Dipartimento di studi in lingua italiana
}

\section{Riassunto}

La presente ricerca esplora la presenza dell'animismo, artificialismo e finalismo nelle conoscenze dei bambini sulla realtà naturale. La ricerca è stata condotta con la partecipazione di 21 bambini di età compresa tra i tre ed i sette anni, chiamati a rispondere alle domande di un'intervista strutturata di tipo piagetiano. Le risposte dei bambini confermano la presenza dei fenomeni indicati, ovvero delle specifiche operazioni mentali, come pure i cambiamenti evolutivi, rilevabili da una significativa diminuzione delle risposte tipicamente animistiche nei bambini più grandi.

Parole chiave: bambini, animismo, artificialismo, finalismo

\section{Introduzione}

Nella prima parte dell'infanzia tutti i bambini sono egocentrici, e questa caratteristica tipica si manifesta nel funzionamento cognitivo, linguistico e affettivo. Il concetto di egocentrismo infantile è stato inizialmente introdotto nell'ambito della psicologia dalla teoria dello sviluppo mentale di Piaget, elaborata in base a numerose osservazioni dirette del comportamento infantile. Nei primi anni di vita il bambino diventa consapevole degli oggetti e delle persone che lo circondano, e le sue scoperte casuali vengono sistematizzate ed assimilate strutturandosi in schemi di azioni. Verso i due anni il bambino inizia a costruire delle teorie sul mondo che lo circonda, e fa ciò mediante il finalismo, l'animismo e l'artificialismo. Questi fenomeni sono, in effetti, delle operazioni mentali specifiche che si trovano alla base del pensiero preoperatorio.

Un esempio tipico di finalismo lo troviamo nella spiegazione che, se la gallina fa 
l'uovo, questo succede perché il bambino possa mangiarlo. L'animismo consiste invece nella convinzione che tutti gli oggetti siano esseri viventi, e dunque il Sole, ad esempio, è cosciente di muoversi e lo fa per dare luce alle nostre attività. L'artificialismo, infine, si manifesta sotto la forma della convinzione che tutto sia stato costruito dalluomo, comprese le montagne, i laghi ed i fiumi.

Per i bambini risulta difficile staccarsi dal proprio punto di vista, e nella loro visione del mondo e delle cose essi sono spesso convinti che gli altri possano percepire i loro pensieri e desideri, anche se non li esprimono verbalmente. Grazie allo sviluppo cognitivo che avviene nel corso del tempo e con la frequentazione di diversi contesti sociali, i bambini incontrano ed acquisiscono nuove regole. In particolar modo, è la scuola dell'infanzia il luogo che offre ai bambini la possibilità di confrontarsi con il mondo degli adulti e con altre autorità all'infuori dei propri genitori. Per di più, nell'interazione con gli altri essi imparano a regolare le proprie emozioni e ad acquisire nuovi schemi di azioni, cosa che lentamente li porta a passare dal proprio punto di vista alla comprensione di quello degli altri.

L'egocentrismo rappresenta la tendenza a trascurare il punto di vista altrui, e anzi la stessa difficoltà di immaginare che un altro soggetto possa percepire il mondo da un punto di vista diverso dal proprio. In un famoso esperimento di Piaget e Inhelder (1967; in Di Stefano 1973), il bambino, posto davanti ad un plastico di una montagna, tendeva ad attribuire il proprio percepito anche ad altre persone poste in punti di vista differenti. In altri contesti il bambino, che doveva spiegare qualcosa ad un altro bambino, trascurava di comunicare informazioni per lui scontate. La fase dell'egocentrismo è tipica della prima infanzia, e si supera attorno ai 7 anni, quando il bambino riesce ad avere un pensiero reversibile e logico di ciò che gli accade intorno. In questa fase il bambino è in grado di condividere con gli altri attività e giochi, è consapevole che le sue azioni hanno delle conseguenze sugli altri, ed è quindi in grado di acquisire delle regole di comportamento socialmente più avanzate.

Il realismo infantile è il primato del materiale anche su fatti mentali (sogni, pensieri, segni, linguaggio verbale). Il bambino è convinto che i nomi delle cose siano contenuti nelle cose e che cambiando il nome ad una cosa questa cambierà parimenti nella sostanza. Per Piaget la conseguenza dell'egocentrismo e del realismo infantile è il pensiero pre-causale. Il pensiero pre-causale è un pensiero "magico", per cui l'erba cresce per far mangiare le mucche, mentre il vento soffia per spostare le nuvole. Nelle interviste piagetiane bambini di 6-7 anni rispondevano che il legno che brucia prova dolore e che il sole cammina per seguire la luna. L'artificialismo infantile è la tendenza 
del bambino ad attribuire anche ad elementi naturali costruzione umana. Così egli può affermare, ad esempio, che una montagna sia stata costruita dai muratori, o che in un tuono qualcuno ha "messo dentro" il fuoco. Per Piaget l'animismo e l'artificialismo infantili sono generati anche dal fatto che il bambino vede assecondato ogni suo bisogno da un adulto. I genitori, visti da un bambino, sono infallibili, e la natura fa parte di un disegno armonico. Da ciò scaturisce il finalismo infantile, ovvero l'idea che la natura segua delle leggi morali.

Nelle ricerche di Piaget i bambini dimostrano una tendenza all'animismo che li porta, fino all'adolescenza, ad attribuire in modo inappropriato caratteristiche animate ad oggetti inanimati. Questa posizione è stata oggetto di annosi dibattiti. Nelle sue ricerche, condotte con bambini del Pacifico del Sud, la Mead (1933; in Dolgin, Behrend, 1984) ha trovato poca evidenza di animismo. In seguito, altri autori riportarono dati a conferma del fatto che l'animismo non risulta tanto presente quanto indicato da Piaget (Huang, Lee, 1945; Klingberg, 1957; in Dolgin, Behrend, 1984). I dati di una ricerca condotta con l'uso di domande che coinvolgevano numerosi esempi appartenenti al mondo animato e inanimato, hanno portato alla conclusione che lanimismo non rappresenta un fenomeno pervasivo (Dolgin, Behrend, 1984). I bambini raramente sbagliavano rispondendo a domande contenenti prototipi animati (umani) ed inanimati, mentre il numero degli errori aumentava quando le domande si riferivano a esempi meno prototipici. Gelman (1978) conclude che esiste un consenso nell'affermare che i bambini non sono tanto animistici quanto inizialmente si era ritenuto, e che le conclusioni precedenti erano probabilmente da attribuirsi piuttosto alle domande poste ai bambini, riguardanti argomenti con i quali essi avevano ben poca dimestichezza.

La presente ricerca prende come punto di riferimento la teoria di Piaget, che è tuttora la teoria stadiale più accreditata nell'àmbito della psicologia dello sviluppo. I principali punti di forza della teoria (Miller, 1996) stanno nel suo riconoscimento del ruolo centrale della cognizione nello sviluppo, nel suo valore come teoria integrativa ed euristica, nella scoperta di caratteristiche sorprendenti del pensiero dei bambini, nella sua vasta portata e nella sua validità ecologica. Le principali debolezze riguardano invece la mancanza di completezza formale, la scarsa attenzione prestata allo sviluppo sociale ed emotivo e le inadeguatezze metodologiche e stilistiche. Malgrado le sue debolezze, essa offre comunque un soddisfacente quadro teorico entro cui esaminare la ricchezza e la complessità dello sviluppo cognitivo. Le proposte interpretative di Piaget sull'egocentrismo infantile e le sue diverse forme di manifestazione e trasformazione continuano tutt'oggi ad essere rilevanti per la nostra comprensione dei bambini (Goncu, Abel, 2010). Una rapida esplorazione della bibliografia recente rivela infatti che i 
ricercatori continuano a cercare evidenze per supportare le sue idee, per rivedere i suoi dati originali e per offrire spiegazioni alternative del suo lavoro (Pramling, 2006).

La ricerca che presentiamo in questo saggio si colloca appunto nel filone degli studi sopra citati, orientati alla ricerca di evidenze e, ove necessario, revisioni delle interpretazioni sul funzionamento del pensiero infantile. Il nostro scopo principale è stato quello di analizzare la presenza di tre fenomeni tipici del pensiero infantile indicati da Piaget: l'animismo, il finalismo e l'artificialismo nelle risposte verbali dei bambini in merito al funzionamento della natura. Considerata l'età dei bambini del nostro campione (da 3 a 7 anni), ipotizziamo che essi si trovino nel periodo preoperatorio ${ }^{[1]} \mathrm{e}$ che dimostreranno, nelle proprie dichiarazioni, caratteristiche tipiche di questo pensiero quali l'animismo, il finalismo e l'artificialismo.

\section{Metodologia della ricerca}

\section{Partecipanti}

Nelle ricerche svolte con bambini più grandi, Piaget di solito impiegava il metodo clinico. Nonostante gli indubbi vantaggi offerti da questo metodo, quali la flessibilità nel poter adattare le domande alla particolare risposta del bambino, tale metodo presenta anche notevoli svantaggi. I due principali, secondo Miller (1996), sono rappresentati dal pericolo che lo sperimentatore possa, con le sue domande, "guidare" eccessivamente il bambino (o, allopposto, guidarlo troppo poco), e che a bambini diversi possano essere poste domande leggermente diverse, mentre - comè ben noto - l'uniformità nelle istruzioni, negli stimoli e nella misura delle risposte è, in psicologia sperimentale, l'elemento fondamentale della garanzia di valore probatorio. Per tale motivo abbiamo deciso di porre a tutti i bambini le stesse domande e di registrare e trascrivere con assoluta fedeltà le loro risposte. Hanno preso parte alle interviste ventuno bambini ${ }^{[2]}$ di età compresa tra i tre ed i sette anni, 12 di genere femminile e 9 di genere maschile.

\footnotetext{
[1] Lesistenza degli stadi è sicuramente una delle questioni teoriche più rilevanti e sulle quali più si è dibattuto. Comunque, oggi tutti (Camaioni, Di Blasio, 2005) riconoscono che lo sviluppo cognitivo procede secondo delle sequenze universali, e si può affermare che Piaget aveva ragione quando parlava di sequenze di sviluppo, pur avendo probabilmente torto nel considerare gli stadi come strutture globali. In questa ottica usiamo il concetto di pensiero preoperatorio.

[2] Le ricerche nelle quali viene usata l'intervista piagetiana, con la conseguente trascrizione e analisi del testo, tipicamente operano su campioni limitati (ad. es. vedi Dolgin, Behrend, 1984; Berzonsky, 1974; de Lima Monteiro et al., 1993).
} 


\section{Strumenti e procedimento}

Ogni bambino è stato intervistato individualmente in merito alle sue credenze sugli astri e sulla natura. L'intervista strutturata era composta come segue ${ }^{[3]}$ :

- Tre domande (più una di conferma) per esplorare l'animismo infantile: Sanno i fiori di venir tagliati? Una barca sa di essere sull'acqua? Il sole e la luna sono vivi? E sanno quando devono sorgere e tramontare ${ }^{[4]}$ ?

- Una domanda per esplorare l'artificialismo: Da dove vengono le pietre delle montagne?

- $\quad$ Una domanda (più una introduttiva) per studiare il finalismo: Il sole si muove ${ }^{[5]}$ ? Perché si muove?

\section{Risultati della ricerca}

Presentiamo in seguito le risposte dei bambini ricevute nel corso della nostra ricerca (le parole dei bambini sono in corsivo, mentre le frequenze figurano nelle parentesi):

Due bambini di 3 e 3,5 anni: Sanno i fiori di venir tagliati? - Sì, sanno e a loro questo fa male. Si, perché quando li prendono muoiono. - Una barca sa di essere sull'acqua? - Si (2). - Il sole e la luna sono vivi? E sanno quando devono sorgere e tramontare? - Sono vivi e sanno quando devono sorgere e tramontare. Si, deve venire perché ho paura della notte. - Il sole si muove? Perché si muove? - Il sole si muove perché è giorno e poi è notte. Si, perché vola nellaria. - Da dove vengono le pietre delle montagne? - Arrivano dal mare. Gli uomini le hanno portate.

Nelle risposte dei bambini di tre anni possiamo notare la presenza dell'animismo, quale tendenza ad attribuire vita, coscienza ed intenzionalità a tutti gli oggetti che si trovano, attivamente o passivamente, coinvolti in qualche processo dinamico (la barca che galleggia sull'acqua, il sole e la luna che si spostano). Evidente pure l'artificialismo infantile, che porta il bambino a pensare che anche certi elementi naturali, come le montagne, siano dovuti all'azione fabbricatrice dell'uomo.

[3] Fonte delle domande: Vianello, 1995.

[4] La domanda serviva per confermare ed eventualmente approfondire quanto dichiarato in risposta alla precedente.

[5] Domanda preparatoria — nell'intervista serviva per attirare l'attenzione del bambino sul fenomeno esplorato. 
Cinque bambini di 4 anni: Sanno i fiori di venir tagliati? - Si (3). No (2). - Una barca sa di essere sull'acqua? - Si (4), perché la barca si muove. No (1). - Il sole e la luna sono vivi? E sanno quando devono sorgere e tramontare? - Sì, perché il sole splende. La luna viene al suo posto di notte. Sono vivi e sanno quando devono venire o andarsene. Si sono su. Sì sanno. Perché il sole splende e dà luce. La luna viene al suo posto di notte e dà la notte. Stanno qui, in cielo! Dio! sì sanno. - Il sole si muove? Perché si muove? - Si (5). Si vede che si muove (2). Non lo trovi nello stesso posto (2), perché dopo il sole deve venire la luna e la notte perché dobbiamo dormire. Non so (2). - Da dove vengono le pietre delle montagne? - Sono sulla montagna (2). Da un signore che porta le pietre sulle montagne. Da per terra. Dal mare.

Rispetto alle risposte che abbiamo rilevato ai tre anni, i bambini di quattro si ripartono in due gruppi, per quanto riguarda la consapevolezza dei fiori: tre bambini affermano infatti l'esistenza della consapevolezza, mentre due la negano. Tutti, comunque, sono concordi per ciò che riguarda gli astri: li considerano vivi, consapevoli del proprio compito ed intenzionati a portare la luce o la notte.

Tre bambini di 5 anni: Sanno i fiori di venir tagliati? - No. No, perché non sono vivi. Si. Una barca sa di essere sull'acqua? - Si. No, (2), perché la barca non è viva. - Il sole e la luna sono vivi? E sanno quando devono sorgere e tramontare? - No (2). Si (1), loro sono vivi e sanno quando devono venire e quando andare via. - Il sole si muove? Perché si muove? - No. Si, perché sorge e tramonta. Perché la terra è rotonda e il sole si deve muovere per dare la luce a tutti sulla terra. - Da dove vengono le pietre delle montagne? - Non lo so. Si creano da sole come i fiori. Dal cielo, le ha creato Dio.

All'età di cinque anni continua la presenza bipolare di opinioni che abbiamo già indicato per l'età precedente in merito alla consapevolezza delle entità "animate" ed inanimate.

Sei bambini di 6 anni: Sanno i fiori di venir tagliati? - No (6). - Una barca sa di essere sull'acqua? - No (6), perché è di papà. Sopra cè il pescatore, mio papà pesca. - Il sole e la luna sono vivi? E sanno quando devono sorgere e tramontare? - No (non sono vivi 3), però sanno quando venire e andare via. Si. Sì. (3) sanno solo quando devono muoversi. Non so perché. Non sanno, perché non hanno gli occhi. - Il sole si muove? Perché si muove? - No (3). Si, perché deve fare luce da tutte le parti (2). Sì, perché viene il giorno. Perché crescono i alberi e i fiori dopo. Non lo so. - Da dove vengono le pietre delle montagne? Non so (3), sono stati fatti. In cima alle montagne. Dalla terra. Certe sono li dà tanto tempo e certe le portano i fiumi. 
A sei anni, tutti i bambini da noi intervistati negano ai fiori la consapevolezza, come pure alla barca, ma sarebbe ancora presto affermare che in questa età l'animismo smetta di esistere, poiché lo ritroviamo ancora, nelle risposte di alcuni, quando l’argomento sono gli astri.

Cinque bambini di 7 anni: Sanno i fiori di venir tagliati? - No, perché non sanno. No (2), perché loro non possono sentire come noi. Loro non sono vivi. - Una barca sa di essere sull'acqua? - Si (1), Si (2) perché si sente bagnata. No, la barca non sente niente (2). - Il sole e la luna sono vivi? E sanno quando devono sorgere e tramontare? - Si, si (2) lo sanno perché il cielo si scurisce arriva la luna e quando si chiarisce arriva il sole. Si, si, perché quando il sole si stanca deve venire la luna per sostituirla. Si, quando il sole vede che la luna se nè andata, arriva lui. No, il sole non è vivo. No, lui solo gira intorno alla terra. - Il sole si muove? Perché si muove? - Si (2), si gira perché se non si girerebbe, noi non saremmo vivi. Perché deve fare il suo lavoro. Un poco si e un poco no. Perché lui deve andare da unialtra parte. Si, pero si muove piano. Non lo so. Si muove perché non può esserci il sole solo da noi, devono vederlo anche quelli che vivono molto lontano da noi. - Da dove vengono le pietre delle montagne? - Dai meteoriti. Dalla montagna. Spuntano fuori delle montagne. Loro sono la da centinaia e centinaia di anni.

Per riassumere e permettere una sintesi di quanto detto abbiamo organizzato i dati nelle tabelle che seguono. Nella Tabella 1 figura il numero di risposte alle tre domande relative alla presenza di vita, coscienza e consapevolezza in entità "animate" (fiori) e inanimate (barca, sole, luna) nelle diverse fasce di età.

Tabella 1. Classificazione delle risposte dei bambini sulla consapevolezza di entità animate e inanimate

\begin{tabular}{|c|cccc|ccc|c|}
\hline & \multicolumn{2}{|c|}{ Fiori } & \multicolumn{2}{c|}{ Barca } & \multicolumn{2}{c|}{ Sole e luna } & \multirow{2}{*}{$\begin{array}{c}\text { Risposte animistiche } \\
\text { / Totale risposte }\end{array}$} \\
\cline { 1 - 6 } Età & Sì & No & Sì & No & Sì & No & $6 / 6$ \\
\hline 3 & 2 & 0 & 2 & 0 & 2 & 0 & $12 / 15$ \\
\hline 5 & 3 & 2 & 4 & 1 & 5 & 0 & $3 / 9$ \\
\hline 6 & 1 & 2 & 1 & 2 & 1 & 2 & $3 / 18$ \\
\hline 7 & 0 & 6 & 0 & 6 & 3 & 3 & $7 / 15$ \\
\hline $\begin{array}{l}\text { Totale } \\
\text { risposte }\end{array}$ & 6 & 15 & 10 & 11 & 15 & 6 & $31 / 63$ \\
\hline
\end{tabular}


Le risposte tipicamente animistiche sono molto frequenti nei più piccoli: si passa infatti dalla totalità di risposte animistiche a tre anni, ad una stragrande maggioranza di esse ai quattro anni, alle quali segue poi una brusca diminuzione nei bambini di 5-6 anni. Ovviamente, è importante prendere in considerazione pure l'argomento esplorato: per i fiori, presi quale esempio di entità animata, l'animismo regredisce subito dopo i 5 anni, mentre rimane anche dopo questo periodo per le entità inanimate quali la barca, il sole e la luna.

Nella Tabella 2 figurano i dati riguardanti l'artificialismo infantile. Rispondendo alla domanda sulle origini delle montagne i bambini coinvolti dalla nostra ricerca hanno usato quattro categorie di risposta: uomo, Dio, natura e 'non so'. Il gruppo più numeroso è quello delle risposte con le quali essi hanno attribuito la creazione delle montagne alla natura (terra, meteoriti, fiumi, mare, le montagne stesse). Quattro bambini su 21 hanno dichiarato di non sapere, due hanno attribuito la creazione alluomo ed uno soltanto a Dio.

Tabella 2. Classificazione delle risposte alla domanda Da dove vengono le pietre delle montagne?

\begin{tabular}{|c|c|c|c|c|}
\hline & uomo & Dio & natura & non so \\
\hline Età & & & & \\
\hline 3 & 1 & & 1 & \\
\hline 4 & 1 & & 4 & \\
\hline 5 & & 1 & 2 & 1 \\
\hline 6 & & & 3 & 3 \\
\hline 7 & & & 6 & \\
\hline Totale $^{[6]}$ & 2 & 1 & 16 & 4 \\
\hline
\end{tabular}

Nella Tabella 3 figura una sintesi delle risposte dei bambini alla domanda volta a studiare il finalismo. Classificare le risposte in questa chiave di lettura (finalistica) risulta alquanto difficile. Le ragioni delle difficoltà incontrate possono essere dovute alla mancanza del fenomeno esplorato; alla compresenza di operazioni mentali (animismo e finalismo), come pure alla necessità di trovare in futuro un altro quadro di interpretazione. Naturalmente, esiste pure la possibilità di mancanza metodologica, ovvero la sbagliata scelta delle domande-stimolo usate per provocare ed esplorare questa forma di operazione mentale. La maggior parte dei bambini (16 su 21) ha risposto alla

[6] Il numero totale delle risposte è superiore a quello dei partecipanti poiché alla domanda sulle origini delle montagne alcuni bambini hanno fornito risposte plurime. 
domanda preparatoria (sul movimento del sole) in modo affermativo, ma per motivi appena elencati non è possibile trarre valide conclusioni a favore dell'esistenza del finalismo.

Tabella 3. Classificazione delle risposte alle domande Il sole si muove? Perché si muove?

\begin{tabular}{|c|c|c|c|c|c|c|}
\hline \multirow{2}{*}{ Età } & \multicolumn{3}{|c|}{ Il sole si muove? } & \multicolumn{3}{|c|}{ Perché si muove? } \\
\hline & Sì & No & Sì e No & $\mathrm{F}$ & Non so & Altro \\
\hline 3 & 2 & & & & & 2 \\
\hline 4 & 5 & & & 1 & 2 & 2 \\
\hline 5 & 2 & 1 & & & 2 & \\
\hline 6 & 3 & 3 & & 2 & 1 & 2 \\
\hline 7 & 4 & & 1 & 4 & 1 & \\
\hline Totale & 16 & 4 & 1 & 7 & 4 & 8 \\
\hline
\end{tabular}

$\mathrm{F}=$ finalismo

\section{Discussione dei risultati}

Nella teoria di Piaget i bambini dai 2 ai 6/7 anni si trovano nello stadio preoperatorio, ed è proprio questa l'età dei bambini che hanno preso parte alla nostra ricerca. La caratteristica principale di questo periodo consiste nella conquista della rappresentazione, che si manifesta come capacità dei bambini di usare simboli, immagini, parole ed azioni che rappresentano altre cose. La capacità del bambino di usare il linguaggio per riferirsi ad oggetti, persone e situazioni, ovvero di usare schemi verbali che descrivono la realtà che si rappresenta mentalmente, è uno dei prerequisiti fondamentali per l'attuazione della ricerca, ragione per la quale non abbiamo coinvolto bambini di età inferiore ai tre anni.

Animismo. Per quanto riguarda lo sviluppo dell'animismo è possibile distinguere quattro periodi diversi (Vianello, 1995). Nel primo, i bambini di età inferiore ai 5-6 anni tendono ad attribuire vita, coscienza ed intenzionalità a tutti gli oggetti coinvolti in qualche forma di processo dinamico (ad esempio la barca, che galleggia sullacqua). Successivamente, nelletà compresa fra i 6 e gli 8 anni, rivelano tendenze animistiche 
solo per le cose in movimento (come ad esempio gli astri, le nuvole, ecc.). Invece, dagli 8 ai 10 anni i bambini distinguono tra gli oggetti che si muovono motu proprio (astri o vento) e quelli che invece si muovono di moto ricevuto, attribuendo vita coscienza ed intenzionalità solo ai primi. Verso gli 11-12 anni le loro concezioni sono simili a quelle degli adulti. Nei brani dei nostri colloqui tenuti con i bambini di età compresa fra $\mathrm{i}$ 3 e 7 anni è possibile notare i primi due livelli nello sviluppo dell'animismo. I nostri dati corroborano le affermazioni di ricercatori come Vianello (1995), il quale afferma che i bambini di età compresa fra i 6 e gli 8 anni circa rivelano tendenze animistiche solo per le cose in movimento. Infatti, i bambini di 6 e 7 anni negano la vita ai fiori, ma continuano a considerare vivi il sole e la luna, trovando spiegazioni diverse: la stanchezza (il sole si stanca), il senso del dovere (quando il sole vede che la luna se në andata, arriva lui; deve fare il suo lavoro). Per quanto riguarda le credenze sugli astri riteniamo interessante menzionare la ricerca condotta da de Lima Monteiro, Batista, Mendes, Rodrigues e Teixeira (1993), che confermano la presenza di tre stadi diversi: nel primo il bambino crede che le stelle sono fatte dall'uomo o da Dio; nel secondo i bambini attribuiscono la creazione all'unione tra la natura e forze artificiali, mentre nel terzo emerge l'origine naturale, a volte in base alla considerazione delle difficoltà materiali. Anche se generalmente l'animismo diminuisce nel corso degli anni, alcune ricerche hanno dimostrato che in alcuni individui esso continua a persistere durante tutto l'arco della vita (ad es. Berzonsky, 1974; e Sheehan, Papalia, 1974; in Billingham, Fu, 1980).

Artificialismo. Per esplorare la presenza dell'artificialismo infantile, inteso quale tendenza a pensare che determinati elementi naturali o fenomeni atmosferici siano dovuti all'azione dell'uomo, abbiamo usato la domanda sulle origini delle montagne. Le trascrizioni delle risposte ottenute figurano nella sezione Risultati ed inoltre i dati sono riportati pure nella Tabella 2. Riassumendo si potrebbe dire che le risposte dei bambini non indicano una forte presenza di artificialismo, ed anzi dimostrano, sotto questo profilo, concezioni simili a quelle degli adulti. A questo punto sembra molto interessante riportare alcune delle risposte alla stessa domanda ottenute da Piaget (in Vianello, 1995): bambino di 5 anni: "Sono dei signori che devono averle portate."; 6 anni: "Con grosse pietre."; 7 anni: "Si prende della terra, poi la si mette sulle montagne, e con questa si fanno delle montagne." Analizzando i brani riportati da Piaget, è possibile notare che non a tutti i bambini venivano poste esattamente le stesse domande - largomento era lo stesso (l'origine delle montagne), ma nel primo caso (bambino di 5 anni) l'autore aveva chiesto: "Da dove vengono le pietre delle montagne?", nel secondo (6 anni) "Come è cominciata quella montagna?" e nell'ultimo (7 anni) "Come sono cominciate le montagne?", per poi approfondire il discorso in base alla prima risposta ottenuta, quasi volesse portare il 
bambino verso la conferma delle proprie attese (ovvero la forza fabbricatrice delluomo). Inoltre, in alcuni casi il ricercatore, con il contenuto stesso della domanda, "indirizzava" già in parte la risposta ("Da dove vengono le pietre delle montagne?"). Nel nostro caso, invece, per evitare le critiche rivolte in questo senso verso il metodo di Piaget, a tutti i bambini è stata posta la stessa domanda, senza ulteriori approfondimenti. Di conseguenza, i nostri dati non indicano una forte presenza dellartificialismo. Ovviamente, i tempi sono cambiati, la metodologia è lievemente diversa ed inoltre esiste sempre la possibilità di trovarci alle prese con nozioni sostanzialmente diverse da quelle del passato. Oltre a ciò, numerose ricerche precedenti documentano che non vi è neppure stabilità $\mathrm{e}$ sistematicità nelle risposte che uno stesso soggetto fornisce a compiti diversi (Camaioni, Di Blasio, 2005), poiché il bambino può trovarsi in una certa fase per quanto riguarda un dato compito e in unaltra per ciò che concerne un compito diverso (e di fatto, abbiamo noi stessi rilevato questo fenomeno nelle risposte dei bambini di sei anni, che negavano l'esistenza della consapevolezza ai fiori e alla barca, mentre la affermavano quando l'argomento erano gli astri).

Finalismo. Il finalismo infantile consiste nell'idea che la natura segua delle leggi morali, ed è stato esplorato attraverso la domanda sul perché il sole si muove. Le risposte alla domanda-stimolo appartengono a tre diverse categorie: risposte tipicamente finalistiche (si gira perché, se no, noi non saremmo vivi; devono vederlo anche quelli che vivono molto lontano da noi); un quarto dei bambini non sapeva rispondere e diceva non lo so, mentre un notevole numero di risposte sono state classificate nella categoria "altro". È appunto questa la categoria più numerosa, e vi troviamo risposte come Perché crescono gli alberi e i fiori. Il pensiero del bambino, in questo periodo, è prelogico ed intuitivo, e per mezzo di esso il bambino ricostruisce le azioni passate ed anticipa quelle ancora da compiere. Nella categoria "altro" troviamo pure risposte descrittive, come ad esempio vola nell'aria, o affermative, come è giorno. Rispondendo a questa domanda i bambini hanno fatto spesso riferimento, in modo diretto o indiretto, al concetto di dovere: si deve muovere per dare la luce a tutti; deve andare da unaltra parte; perché dopo il sole deve venire la luna e la notte; perché dobbiamo dormire. Dunque, in questo senso i bambini si appellavano a doveri universali e all'uguaglianza per tutti: Si muove perché non può esserci il sole solo da noi, devono vederlo anche quelli che vivono molto lontano da noi.

Egocentrismo. Interessanti pure gli esempi di tendenze egocentriche che portano il bambino a credere che il sole gira perché se non si girasse, noi non saremmo vivi; oppure deve venire perché ho paura della notte, e dunque mettono l'esistenza degli esseri umani al centro della ragione di esistere degli astri. Anche se la nostra indagine non mirava in modo diretto alla ricerca dell'egocentrismo, esso emerge comunque nelle 
risposte verbali ottenute dai bambini e perciò riteniamo significativo segnalarlo.

Secondo Piaget l'artificialismo e l'animismo sono prodotti da più fattori, tra i quali troviamo il fatto che fin dalla nascita il bambino vive seguito da persone che si occupano in modo intenso di lui e dei suoi bisogni e desideri. Ciò può di conseguenza portare il bambino a pensare che i genitori (o gli adulti in generale) siano in grado di risolvere nello stesso modo ogni problema, compreso quello relativo alla formazione (intesa come "fabbricazione") delle montagne. Ad ogni modo, l'artificialismo e l'animismo sono, almeno all'inizio, fenomeni complementari, e si uniscono a formare il pensiero infantile.

\section{Conclusioni}

Nonostante le numerose critiche ad essa rivolte, la teoria di Piaget continua a rimanere un punto fermo per la comprensione dello sviluppo mentale del bambino. I dati della nostra ricerca confermano l'esistenza di operazioni mentali specifiche nel pensiero preoperatorio dei bambini, e rappresentano un tentativo di fornire dati statistici sui fenomeni esplorati quale supporto alle nude e a volte soggettive interpretazioni di protocolli (uno dei motivi per il quale l'Autore svizzero è stato spesso criticato). Attraverso l'esame della letteratura riguardante i vari aspetti e concetti della teoria di Piaget, è possibile cogliere gli sforzi degli studiosi per fornire approfondimenti e dati di conferma alle interpretazioni originarie, e se necessario, offrire spiegazioni alternative sul funzionamento e sullo sviluppo mentale dei bambini. 


\section{BIBLIOGRAFIA}

BILLINGHAM, R. E., FU, V. R., 1980, Animistic thinking between parents and children, in The Journal of Psychology, 105, 35-39.

BERZONSKY, M. D., 1974, Reflectivity, internality, and animistic thinking, in Child Development, 45, 785-789.

CAMAIONI, 1., DI BLASIO, P., 2005, Psicologia dello sviluppo, il Mulino, Bologna.

DE LimA MONTEIRO, M. T., BATISTA, S. M., MENDES, S. M. P., RODRIGUES, E. C., TEIXEIRA, E., 1993, The child's view of the world: the origin of moon, sun and stars, in Paper presented at the Biennial Meeting of the International Society for the study of behavioral development, Brazil, 1-7.

DI STEFANO, G., (a cura di), 1973, Lo sviluppo cognitivo, Giunti, Firenze.

DOLGIN, K. G., BEHREND, D. A., 1984, Children's knowledge about animates and inanimate, in Child Development, 55, 1646-1650.

GELMAN, R., 1978, Cognitive development, in Annual Review of Psychology, 29, 297-332.

GONCU, A., ABEL, B., 2010, The child's conception of the world: a 20th-century classic of child psychology, in Infant and Child Development, 20, 246-248.

MILLER, P. H., 1996, Teorie dello sviluppo psicologico, il Mulino, Bologna.

PRAMLING, N., 2006, " The clouds are alive because they fly in the air as if they were birds": A re-analysis of what children say and mean in clinical interviews in the work of Jean Piaget, in European Journal of Psychology and Education, XXI (4), 453-466.

VIANELLO, R., 1995, Psicologia dello sviluppo. Edizioni Junior, Bergamo. 


\section{ANIMIZAM, ARTIFICIJALIZAM I FINALIZAM U PREDOPERATIVNOME MIŠLJENJU}

Ovim se istraživanjem provjerava prisutnost animizma, artificijalizma i finalizma u dječjim spoznajama o prirodi. U istraživanju je sudjelovalo 21 dijete od tri do sedam godina, a primijenjen je strukturirani intervju piagetova tipa. Odgovori djece potvrđuju prisutnost istraživanih fenomena, odnosno specifičnih mentalnih operacija, kao i razvojne promjene u vidu smanjivanja tipično animističkih odgovora u starije djece u odnosu na mlađu.

Ključne riječi: djeca, animizam, artificijalizam, finalizam

\section{ANIMISM, ARTIFICIALISIM AND FINALISM IN PRE-OPERATIVE THINKING}

The aim of this work was to explore the existence of animism, artificialism and finalism in the knowledge of children regarding nature. Twenty one children aged between three and seven took part in this study, conducted by means of a Piagetian structured interview. The children's responses confirm the presence of explored specific mental operations, as well as a developmental change in the form of a reduced presence of animistic interpretation in older children.

Key words: children, animism, artificialism, finalism 